\title{
Exploring the Mechanism of Resveratrol in Reducing the Soft Tissue Damage of Osteoarthritis Based on Network Pharmacology and Experimental Pharmacology
}

\author{
Zhiyong Long, ${ }^{1}$ Wang Xiang, ${ }^{2}$ Jun Li $\mathbb{D},{ }^{3}$ Tiejun Yang $\mathbb{D}^{,},{ }^{3}$ and Ganpeng Yu $\mathbb{D}^{3}$ \\ ${ }^{1}$ Shantou University Medical College, Shantou University, Shantou, Guangdong, China \\ ${ }^{2}$ The Affiliated Hospital of Guilin Medical University, Guilin, Guangxi Province, China \\ ${ }^{3}$ People's Hospital of Ningxiang City, Ningxiang City, Hunan Province, China \\ Correspondence should be addressed to Jun Li; lijun.guke@hotmail.com, Tiejun Yang; yangtiejun321@outlook.com, and \\ Ganpeng Yu; yuganpeng.guke@hotmail.com
}

Received 6 March 2021; Revised 25 July 2021; Accepted 8 September 2021; Published 4 October 2021

Academic Editor: Jun Jiang

Copyright ( 12021 Zhiyong Long et al. This is an open access article distributed under the Creative Commons Attribution License, which permits unrestricted use, distribution, and reproduction in any medium, provided the original work is properly cited.

\begin{abstract}
Aim. To explore the mechanism of resveratrol in reducing the soft tissue damage of osteoarthritis (OA) based on network pharmacology. Methods. Pharmmapper was used to predict the target of resveratrol, OMIM and Genecards were used to collect OA-related disease genes, and David ver 6.8 was used for enrichment analysis. Then, animal experiments were carried out for verification. The rat $\mathrm{OA}$ model was established and the rats were randomly divided into 4 groups: model group, resveratrol lowdose group, resveratrol high-dose group, and blank control group for follow-up experiments. Hematoxylin-eosin (HE) staining was used to detect the degree of pathological damage of rat bones and joints. Enzyme-linked immunosorbent assay (ELISA) was used for the content of inflammatory factors. Western blot was used to detect the expression of Toll-like receptor 4 (TLR4), Myeloid differentiation factor 88 (MyD88), nuclear factor kappa B protein (NF- $\kappa$ B), cysteine protease-9 (CASP-9), Bcl-2 protein, and Bax protein. Results. Through network pharmacological analysis, this study found that resveratrol may regulate the TLR4 signaling pathway, PI3K-Akt signaling pathway, FoxO signaling pathway, Osteoclast differentiation, Rheumatoid arthritis, etc. Animal experiments showed that compared with the model group, the pathological damage of bone and joint in the resveratrol low-dose and high-dose groups was significantly improved. Compared with the model group, the serum levels of IL-1beta, IL-6, IL-17, TNF- $\alpha$, and MCP-1 in the resveratrol low-dose and high-dose groups were significantly reduced $(P<0.05)$; protein levels of TLR-4, MyD88, and NF- $\kappa$ B p65 were significantly reduced $(P<0.05)$; caspase- 9 and Bax protein levels were significantly reduced $(P<0.05)$, and $\mathrm{Bcl}-2$ was significantly increased $(P<0.05)$. Conclusion. Resveratrol may inhibit the activation of the TLR4mediated NF- $\kappa$ B signaling pathway and has a repairing effect on soft tissue damage in OA.
\end{abstract}

\section{Introduction}

Osteoarthritis $(\mathrm{OA})$ is a degenerative joint disease characterized by progressive articular cartilage destruction, subchondral bone changes, osteophyte formation, and synovial inflammation [1]. The clinical symptoms are mainly joint pain, swelling, stiffness, and mobility disorders, which seriously affect the quality of life of patients [2-4]. In the past 20 years, the number of patients with $\mathrm{OA}$ has increased dramatically $[5,6]$. It is estimated that the current prevalence of $\mathrm{OA}$ in people over 50 years old worldwide is as high as
$10 \%$ to $20 \%$, and in the next 30 years, its prevalence may double [7]. So far, the clinical treatment of OA is mainly to relieve pain and maintain joint function. At present, nonsteroidal anti-inflammatory drugs (NSAIDs) have been used as a routine treatment for OA, but their adverse events should not be underestimated [8]. Therefore, it is very important to explore the occurrence and development mechanism of OA and find safer and more effective treatments for the prevention and treatment of OA.

Resveratrol is a natural polyphenol plant compound with a symmetric diphenylethylene structure. Its content is high 
in the rhizomes of grapes, cranberries, peanuts, mulberries, and other plants and the traditional Chinese medicine Polygonum cuspidatum [9-11]. It has almost no toxic and side effects on the human body and has been proven to have anti-inflammatory, antitumor, antioxidant, and immuneregulating effects [12]. Studies have shown that resveratrol has anti-inflammatory and chondroprotective effects in a rabbit OA model induced by endotoxin (LPS) [13]. Hua et al. found that resveratrol can prevent sodium nitrosoferricyanide-induced OA-like inflammation [14]. However, the mechanism of resveratrol in the treatment of $\mathrm{OA}$ is still unknown.

As a new interdisciplinary developed in recent years, network pharmacology has changed the previous drug discovery model of "single gene, single target, single disease." The emergence of network pharmacology is of great significance for the discovery of new drugs and the multitarget research of drugs, and it is mainly used for drug toxicity prediction and drug readjustment indications $[15,16]$. For different kinds of diseases, the location and characteristics of drug targets and disease genes in the network are different. Research shows that the distance between the drug target and the key node of the disease network has certain characteristics in the network. In addition, studies have found that in the process of rational drug design, the network distance between drug targets and corresponding disease genes has a tendency to become smaller and smaller [17]. This suggests that it is of great significance to probe the characteristics of drug targets in biological networks. Therefore, this study would explore the molecular network of resveratrol in the treatment of OA through network pharmacology and further verify the related signaling pathways and biological processes in OA rat models. The idea and process of this research are shown in Figure 1.

\section{Materials and Methods}

2.1. Potential Targets of Resveratrol Prediction and OA Disease Gene Collection. The relevant chemical information and several potential targets of resveratrol were searched through the PubChem database (https://www.ncbi.nlm.nih.gov) [18], and the chemical structure of resveratrol was drawn using ChemDraw 3D software and saved in sdf format. The SMILES structure of resveratrol was also collected from Pubchem. The "sdf" format of resveratrol was input into Pharmmapper (http://lilab-ecust.cn/pharmmapper/) to predict its potential targets [19]. The SIMES structure of resveratrol was input into Swiss Target Prediction (http:// www.swisstargetprediction.ch/) [20], Similarity ensemble approach (SEA) (http://sea.bkslab.org/) [21], and STITCH Database (http://stitch.embl.de/) [22] to obtain the potential targets. The resveratrol targets were imported into UniProt (http://www.uniprot.org/) to obtain the official gene symbol. Finally, those targets were combined and deduplicated to obtain the set of resveratrol potential targets.

OA disease genes were searched and collected using the OMIM database (http://omim.org/) and Genecards (http:// www.genecards.org) $[23,24]$. The search results of Genecards and OMIM were merged and deduplicated to obtain
OA-related genes set. The resveratrol targets and OA genes are shown in Table S1 (see supplementary materials). The potential target set of resveratrol and the OA target set were compared, and the overlapping part of the two was considered as the target of resveratrol in the treatment of OA (Table S2).

2.2. Resveratrol-OA Protein-Protein Interaction (PPI) Network Construction and Analysis. The String database (https://string-db.org/) is a database for searching protein interactions, including both direct physical interactions between proteins and indirect functional correlations [25]. The targets were input into String to collect the PPI data. Cytoscape 3.7.1 software was utilized for visualization. The node degree and betweenness centrality are used to reflect the importance of the node. The larger the value of the degree and the betweenness centrality, the more important the node in the network.

In order to further understand the functions of core target genes and the main pathways of resveratrol in the treatment of OA, the resveratrol-OA targets in the resveratrol-OA PPI network were input into David database (https://david.ncifcrf.gov/home.jsp) for Gene Ontology (GO) enrichment analysis and KEGG pathway enrichment analysis, and the species was selected as "Homo sapiens" [26].

2.3. Experimental Animals. Eighty (80) 6-week-old, SD male healthy rats were purchased from Guangdong Experimental Animal Center and raised in a standard environment, license number SCXK (Guangdong) 2018-0016. The weight of the rat is $(180 \pm 10) \mathrm{g}$. The rats were kept in a temperaturecontrolled $\left(22^{\circ} \mathrm{C} \pm 1^{\circ} \mathrm{C}\right)$ and light-controlled animal facility (200 lux, 12-hour light-dark cycle) for 7 days. The experiment was approved by the Animal Ethics Committee of Shantou University Medical College.

2.4. Reagents and Instruments. Resveratrol was purchased from the National Institute for the Control of Pharmaceutical and Biological Products (batch number: 110742200517, purity 99.9\%). Collagenase Type II, (Cat. No.: C2-28, sigma company); BCA protein quantitative kit (batch number Q1220551), phenylmethylsulfonyl fluoride (PMSF, batch number: RE2173411), ethyl iodide Amide (IAA, batch number QL224490), RIPA high-efficiency lysate (batch number TJ272371), Ammonium bicarbonate powder (batch number BCBN6056 V) were purchased from Sigma Inc. The PVDF membrane was purchased from Millipore, USA (Cat. No. IPVH00010). Trypsin-EDTA digestion solution was purchased from Jiangsu KGI Biotechnology Co., Ltd. (Cat. No. KGY0012). HRP goat anti-rabbit secondary antibody IgG (Cat. No. ZB 2306) was purchased from Beijing Zhongshan Jinqiao Biotechnology Co., Ltd. The chemiluminescent substrate (Cat. No. A38555) was purchased from Thermo Company. IL-1 $\beta$ (H002), IL-6 (H007), IL -17 (H014), TNF- $\alpha$ (H052), and MCP-1 (H115) ELISA kits were purchased from Nanjing Jiancheng Institute of 


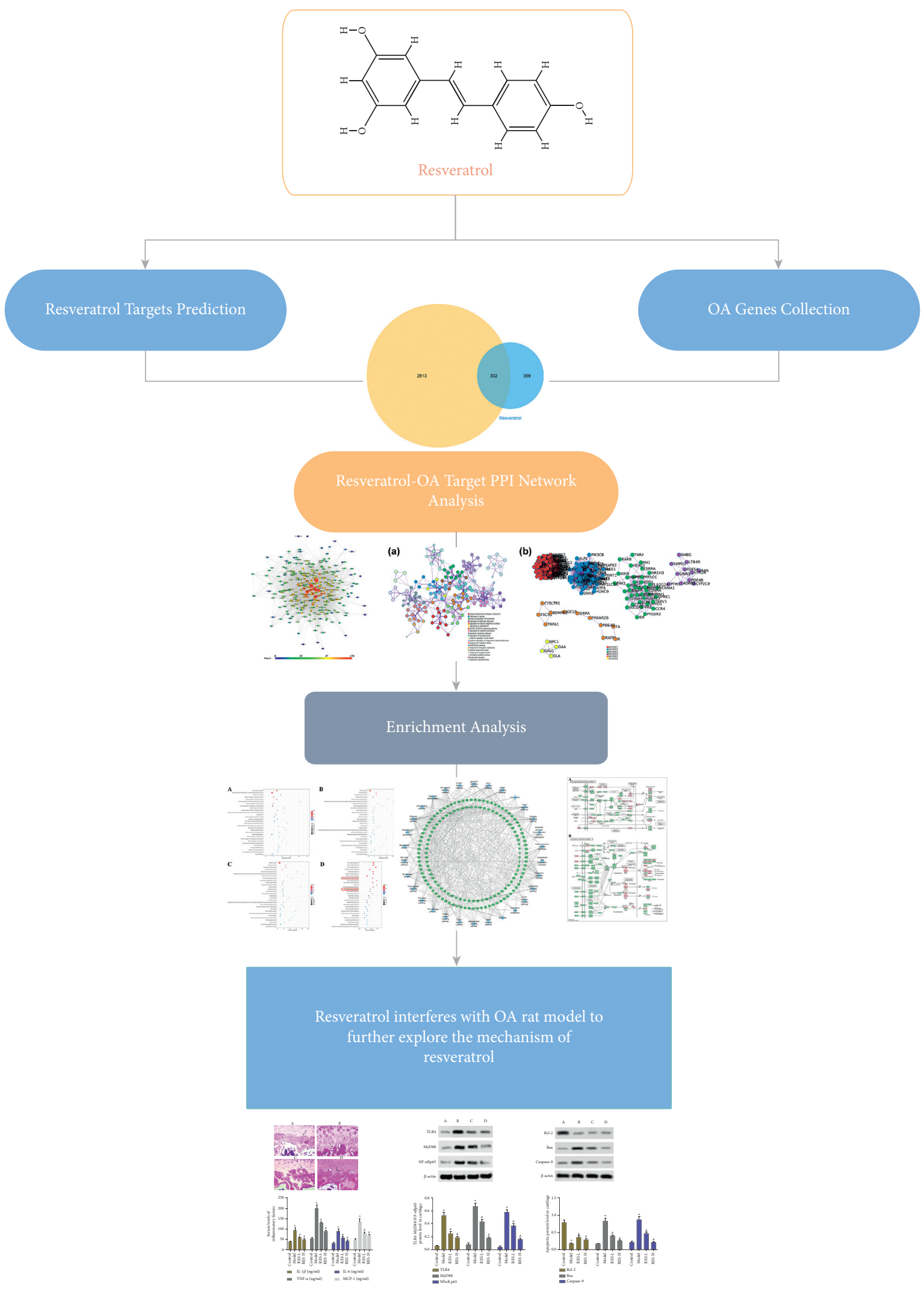

Figure 1: The idea and process of this research. 
Bioengineering. Antibody TLR-4 (BA1717) and Antibody MyD88 (A00025-1) were purchased from Boster Biological Technology Co, Ltd. Antibody NFאB p65 (ATA33904) was purchased from AtaGenix (Wuhan) Technology Co., Ltd. Antibody Caspase-9 (ab32539), antibody Bcl -2 (ab182858), antibody $\mathrm{Bax}(\mathrm{ab} 32503)$, and antibody $\beta$-actin (ab8226) were purchased from Abcam Inc. The desktop high-speed refrigerated centrifuge was purchased from Sigma Inc. The microplate reader was purchased from Thermo Company. The tissue disrupter was purchased from SPEX SamplePrep.

2.5. Animal Modeling, Grouping, and Intervention. The rats were randomly divided into a model group, resveratrol lowdose group, resveratrol high-dose group, and blank control group, with 20 rats in each group. The rats in the model group, resveratrol low-dose group, and resveratrol highdose group were modeled [27, 28].

After the rats were anesthetized with 3\% sodium pentobarbital $(40 \mathrm{mg} / \mathrm{kg})$, the right knee joint was disinfected with iodophor 3 times, and then the knee joint was punctured with a $1 \mathrm{~mL}$ syringe. $50 \mu \mathrm{L}$ of Type II collagenase ( 425 $\mathrm{U} / \mathrm{mg}$ ) with a concentration of $4 \mathrm{mg} / \mathrm{mL}$ was injected into the joint cavity. No other special treatment was given after the operation. On the 4th day, the above operation was repeated once, and the animals were raised freely for 3 days after making the model. The rats in the control group were injected with the same volume of normal saline at the same place and time point.

After the model was completed, the drug was started after 3 days of free breeding. Resveratrol low-dose group was given resveratrol $40 \mathrm{mg} / \mathrm{kg}$ and the resveratrol high-dose group was given resveratrol $80 \mathrm{mg} / \mathrm{kg}$ by gavage, twice daily for 4 weeks. The control group and the model group were given the same amount of normal saline intragastrically.

\subsection{Collection, Preparation, and Observation of Knee Joint} Specimens. After 4 weeks of intervention, the rats were fasted for 10 hours. After the rats were anesthetized by $3 \%$ sodium pentobarbital $(40 \mathrm{mg} / \mathrm{kg})$ intraperitoneal injection, about $4 \mathrm{~mL}$ of blood was taken from the abdominal aorta and placed in a $5 \mathrm{~mL}$ heparin sodium anticoagulation tube. After the blood was taken, the rats were sacrificed by neck dislocation. The entire knee joint was dissected and fixed with $4 \%$ paraformaldehyde for $24 \mathrm{~h}$ and decalcified with $15 \%$ ethylenediaminetetraacetic acid (EDTA) for 4 weeks. The cartilage was cut along the coronal surface of the joint, dehydrated by ethanol, transparent in xylene, embedded in paraffin, and sectioned. The thickness of the section was $7 \mu \mathrm{m}$. The isolated cartilage tissue is used to detect protein inflammation and apoptotic protein expression by the Western Bolt method.

2.7. Pathological Observation. The articular cartilage sections were deparaffinized in xylene, then hydrated with gradient alcohol, and stained according to the instructions of the HE staining kit. The sections were stained in hematoxylin for $5 \mathrm{~min}$, dyed in eosin staining solution for $2 \mathrm{~min}$, soaked in distilled water, and then dehydrated in gradient alcohol in turn. Finally, it was made into pathological sections.

2.8. Detection of Serum Inflammatory Factors by ELISA. The rat blood was centrifuged at 3,000 r/min for $10 \mathrm{~min}$, and the supernatant was taken. The antigen is dissolved in $50 \mathrm{mmol} / \mathrm{L}$ carbonate coating buffer at $4^{\circ} \mathrm{C}, 100 \mu \mathrm{L} /$ well is transferred to a 96-well microtiter plate, the antigen is coated overnight, and the coating solution is discarded. After washing, each well was blocked with $150 \mu \mathrm{L}$ of $1 \%$ BSA for 1 hour, washed with PBST 3 times, $100 \mu \mathrm{L}$ of serum with different dilution ratios was added, and control samples were added and incubated at $37^{\circ} \mathrm{C}$ for 2 hours. Then it was washed 5 times with PBST, $100 \mu \mathrm{L}$ of diluted HRP-labeled secondary antibody was added and incubated at $37^{\circ} \mathrm{C}$ for $1 \mathrm{~h}$. After washing 5 times with PBST, the color developer was added to develop color for $20 \mathrm{~min}$ and measured with a microplate reader.

\subsection{Detection of Inflammatory Factors by Western Blot.} The rat cartilage tissue was prepared as a tissue homogenate, dissolved on ice for $25 \mathrm{~min}$, centrifuged at $12,000 \mathrm{r} / \mathrm{min}$ for $10 \mathrm{~min}$, and cell lysate containing protease inhibitors was added for total protein extraction. The BCA kit was used to determine protein content. An equal amount of protein sample $(20 \mathrm{mg})$ was extracted and denatured at $100^{\circ} \mathrm{C}$ for $5 \mathrm{~min}$. The proteins were then separated using SDS-PAGE gel electrophoresis and transferred to PVDF membranes. At $4^{\circ} \mathrm{C}$, the PVDF membrane was added with corresponding monoclonal primary antibodies [TLR4 $(1: 200)$, MYD88 (1: 500), NF-KBp65 (1:500), Caspase-9 (1:100), Bcl- 2, Bax (1: $100), \beta$-actin $(1: 500)]$ and incubated overnight. Then the horseradish peroxidase-labeled secondary antibody (1:2 000) was added at $4^{\circ} \mathrm{C}$. The color was developed by the chemical substrate luminescence method. ChemiDoc XRS + System gel imager was used for image scanning analysis, ImageQuaNT software was used to measure its absorbance, and each $\beta$-actin was used as an internal reference to analyze the relative expression level.

2.10. Statistical Analysis. All the experimental data are statistically analyzed with the statistical software SPSS 20.0. The experimental results are expressed as mean \pm standard deviation. An Independent $t$-test was used for pairwise comparison, and differences between groups were tested by one-way analysis of variance.

\section{Results and Discussion}

3.1. Resveratrol-OA PPI Network Analysis. A total of 671 resveratrol potential targets and $3114 \mathrm{OA}$ genes (3114 genes were searched from Genecards and 42 were searched from OMIM) were obtained. There are a total of 302 common targets in the resveratrol potential target set and OA gene set, which are considered as potential targets for resveratrol to treat OA. Among the 302 Resveratrol-OA targets, 297 can 
interact with each other. Hence, these 297 were used to construct the Resveratrol-OA PPI network (Figure 2).

3.2. Enrichment Analysis of Resveratrol-OA Targets. The 297 Resveratrol-OA targets in Resveratrol-OA targets PPI network were input into David for enrichment analysis. In the enrichment results of $\mathrm{FDR}<0.05$, the biological processes, cell components, molecular functions, and signal pathways that may be related to OA were screened. Finally, a total of 171 OA-related biological processes, 43 cell components, 90 molecular functions, and 30 signal pathways were obtained (Table S3 and Figure 3). The top 30 biological processes, cell components, molecular functions, and signaling pathways are shown in Figure 4. The relationship between signaling pathways and targets is shown in Figure 5. The targets and genes in the Toll-like receptor signaling pathway and NF-kB signaling pathway are shown in Figure 6 as an example (the Resveratrol-OA targets were marked in pink) [29].

$\mathrm{OA}$ is a common degenerative disease that plagues middle-aged and elderly people. The degeneration of articular cartilage caused by the degradation of the cartilage extracellular matrix is the main pathological change of osteoarthritis. The extracellular matrix is mainly composed of type II collagen and Aggrecan. Mature chondrocytes can synthesize and secrete extracellular matrix, which plays a key role in maintaining the dynamic balance between extracellular matrix anabolic and catabolism [30]. Matrix metalloproteinases (MMPs) play an important role in the degeneration of osteoarthritis. MMP-13 is the most effective type II collagen degrading enzyme [31]. In OA pathological process, the secretion of MMP-13 increases and destroys Aggrecan and type II collagen in the extracellular matrix, which ultimately leads to cartilage degeneration and destruction [32].

In the OA model induced by medial meniscus instability surgery, MMP-13 knockout mice inhibited the development of OA by protecting cartilage from proteoglycan loss and structural damage [33]. In clinical samples, MMP-13 is abnormally expressed at different stages of OA: it is upregulated in the cartilage of patients with OA in the early stage and downregulated in the late stage [34]. In addition, MMP13 is a central node in the cartilage degradation network [35], and its activity can be regulated at multiple levels such as transcription, epigenetic changes, and autophagy [36, 37]. The intervention of resveratrol can decrease the expression of MMP-13, Nuclear factor kappaB (NF-kB) and other inflammatory factors closely related to $\mathrm{OA}$, such as Interleukin-6 (IL-6), Cyclo-oxygenase-2 (COX-2) [38], IL-1 $\beta$ [39], etc. However, the mechanism of resveratrol's anti-OA effect has not been elucidated. Studies have shown that OA may be related to chronic low-grade inflammation, and the expression of a variety of cytokines and inflammatory mediators in the OA state is significantly increased [40], and these inflammatory responses may be related to TLR4. TLR4 can induce synovial cells, chondrocytes, etc., to secrete and release IL- $1 \beta$ and other inflammatory factors, which play a key role in the pathogenesis of OA. The study also found that the expression of TLR4 in OA chondrocytes induced by
IL-1 $\beta$ increased significantly, but after treatment with resveratrol, its expression decreased significantly. Therefore, it is believed that the occurrence and development of OA may be related to the activation of the TLR4 signaling pathway [41], and resveratrol can exert anti-OA effects by inhibiting the Toll-like (TLR4) signaling pathway [42].

TLR4 is one of the important members of the TLRs family and plays a vital role in the process of inducing inflammation [38]. At present, studies have shown that resveratrol can exert anti-OA effects by inhibiting TLR4/MyD88-dependent and independent signaling pathways [43]. Other studies have shown that resveratrol can also exert anti-inflammatory effects through the PI3K/Akt signaling pathway in macrophages [44]. $\mathrm{PI} 3 \mathrm{~K}$ is an important member of the phospholipid kinase family $[45,46]$. Akt is an important direct downstream molecule of PI3K, and activation of PI3K can directly promote Akt phosphorylation and activation. The activation or inhibition of Akt can directly act on its downstream signal molecules and then play a role in regulating cell proliferation, apoptosis, or other important physiological processes [47]. It has been reported in the literature that when LPS acts on human pancreatic cancer cells, it can upregulate the expression of PI3K and Akt. But after adding TLR4 siRNA to silence TLR4, the expression of TLR4 and p-Akt decreased significantly, and after silencing TLR4 and then stimulated by LPS, the expression of TLR4 and p-Akt was still lower than that of the simple LPS group [48]. Xu et al. found that, compared with the hypoxia group, the expression of TNF-a, IL- 6 , and IL- $1 \beta$ mRNA in the group treated with resveratrol was significantly reduced compared with the hypoxia group. After resveratrol treatment or using LY294002 to inhibit PI3K, the expression of p-Akt decreased significantly. This shows that resveratrol can protect pulmonary artery smooth muscle cells by inhibiting the PI3K/ Akt signaling pathway [49]. However, studies have also shown that resveratrol can increase the protein expression of p-Akt in vascular smooth muscle cells while inhibiting the expression of inflammatory factors, that is, by activating the PI3K/Akt signaling pathway to protect vascular smooth muscle [50]. Zong et al. found that when LPS acts on RAW 264.7 cells, the protein expression of p-Akt increases and the PI3K/Akt signaling pathway is activated. Under the combined action of resveratrol and LPS, the activation of the PI3K/Akt signaling pathway was more significant, and the protein expression of $\mathrm{p}$-Akt was further higher than that of the LPS group [51]. It shows that Veratrol may play an anti-LPS-induced inflammatory response in RAW 264.7 cells by activating the PI3K/Akt signaling pathway. The systematic pharmacology part of this study shows that resveratrol can regulate inflammation pathways such as PI3K/Akt signaling pathway, NK-KB signaling pathway, and TNF- $\alpha$ signaling pathway.

3.3. Pathological Changes. The surface of the articular cartilage of the blank control group is flat, the cartilage structure and tidemark of 4 layers are clear, and the chondrocytes are arranged neatly. The cartilage surface in the model group is irregular, cracks are generated, the tidemark recognition is poor, the cartilage structure of 4 layers is unclear, and the cartilage cells proliferate. The cartilage surface of the 


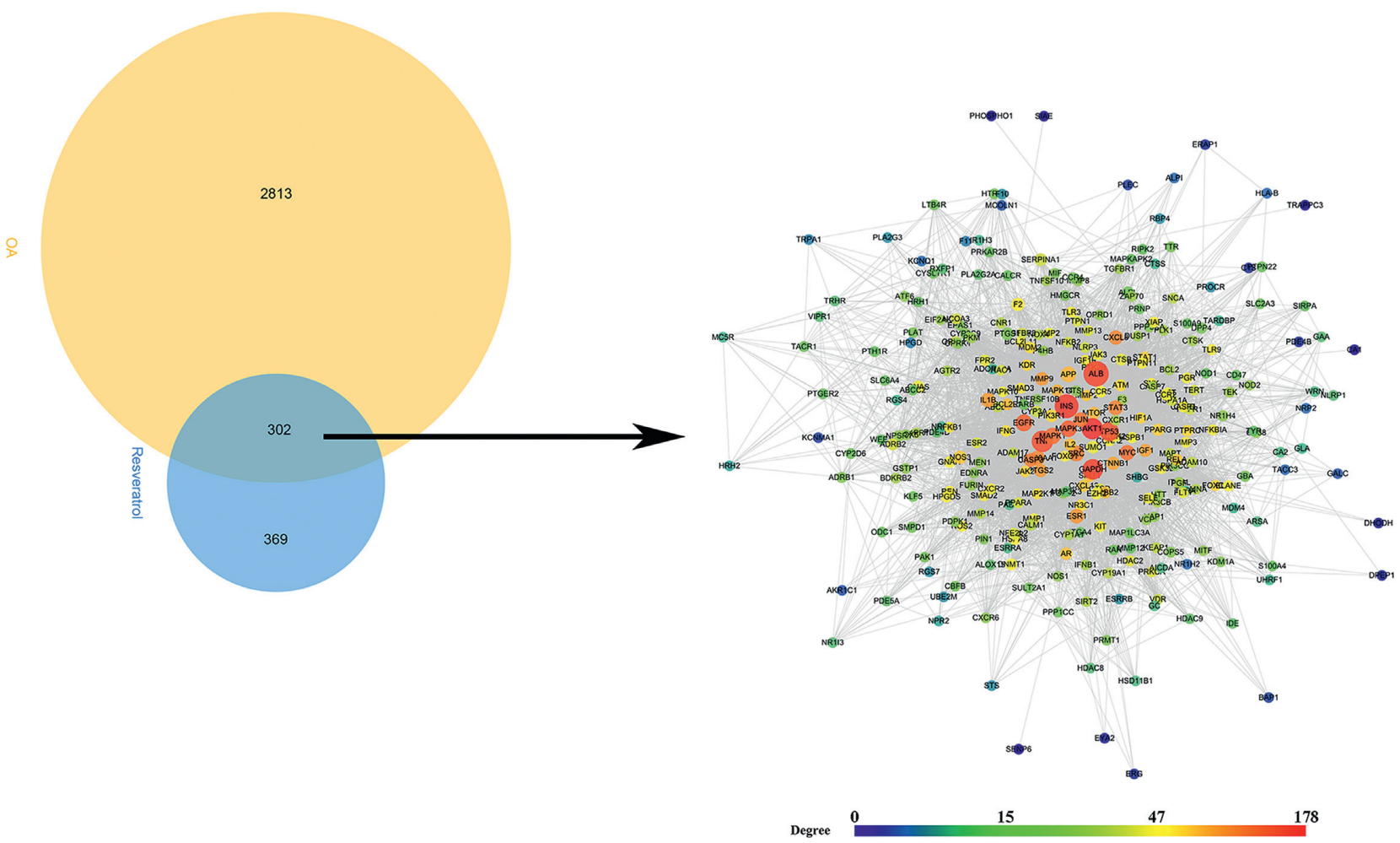

FIGURe 2: Venn diagram and Resveratrol-OA PPI network (the size of the node is positively correlated with its betweenness centrality.).

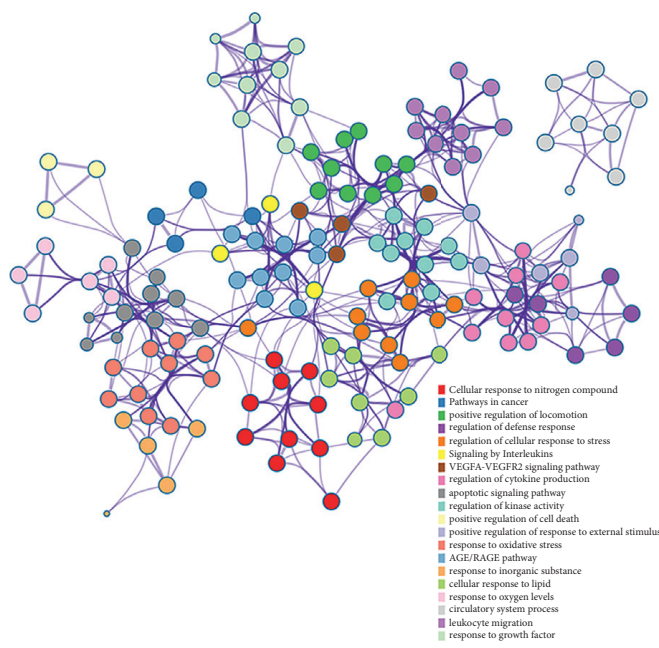

(a)

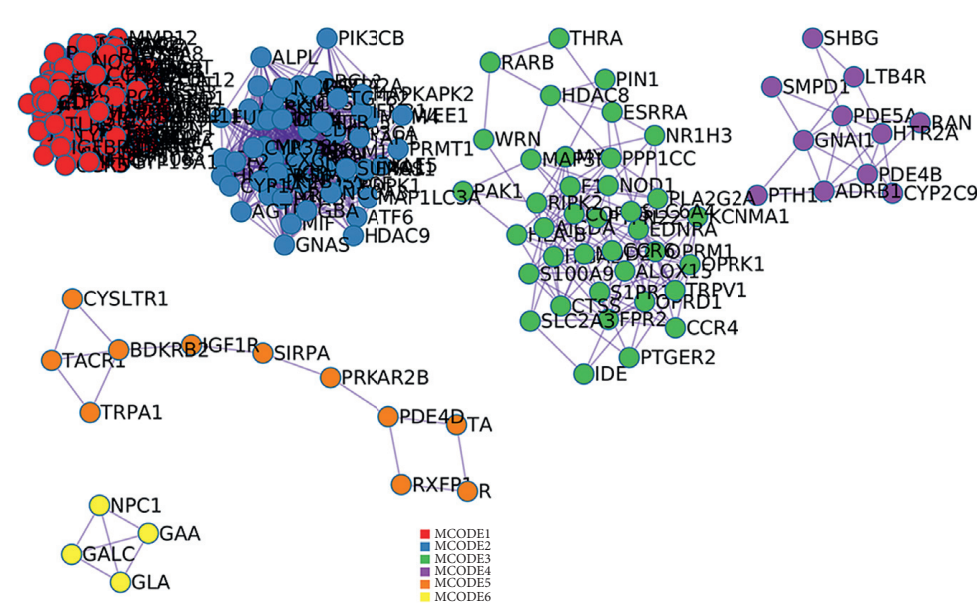

(b)

FIGURE 3: Visualizations of functional enrichment and interactome analysis result. (a) PPI network colored by cluster; (b) clusters.

resveratrol low-dose and high-dose groups was more regular, cracks were reduced, a small amount of chondrocyte proliferation was seen, and the tidemark was visible. Compared with the model group, the degree of cartilage pathological damage was reduced (Figure 7).

3.4. Effect of Resveratrol on Serum IL-1 $\beta, I L-6, T N F-\alpha$, and $M C P-1$ Content. Compared with the control group, the levels of IL- $1 \beta$, IL- 6 , TNF- $\alpha$, and MCP- 1 in the model group were significantly increased $(P<0.05)$. Compared with the model group, the levels of IL- $1 \beta$, IL- 6 , TNF- $\alpha$, and MCP- 1 in the resveratrol low-dose and high-dose groups were significantly reduced $(P<0.05)$ (Figure 8$)$.

3.5. Effect of Resveratrol on the Expression of TLR-4, MyD88, and NF-Kb p65 Protein. The expression levels of TLR-4, MyD88, and NF- $\kappa \mathrm{B}$ p65 protein in rat cartilage tissue were performed by Western blot. Compared with the control group, the expression of TLR-4, MyD88 and the ratio of NF$\kappa \mathrm{B}$ p65 in the model group were significantly increased 


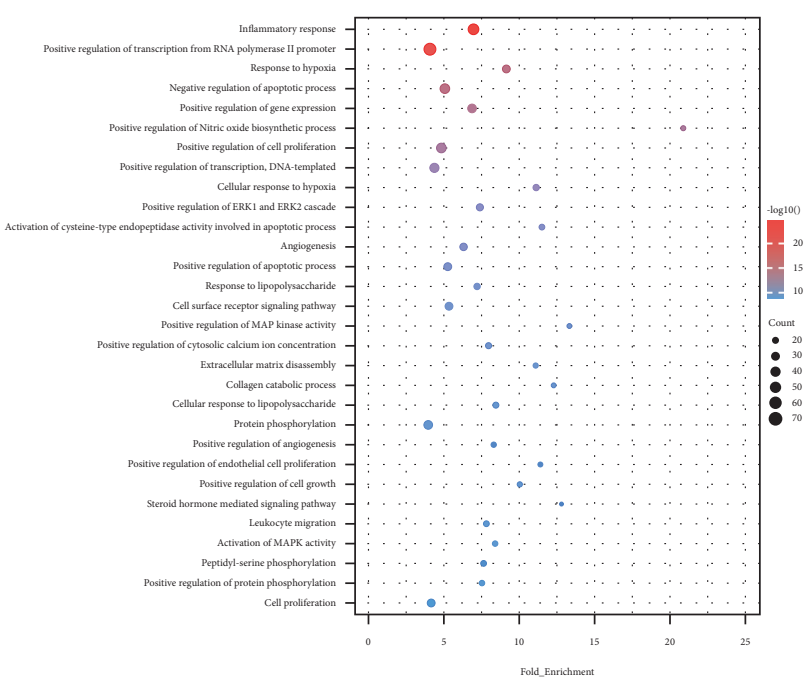

(a)

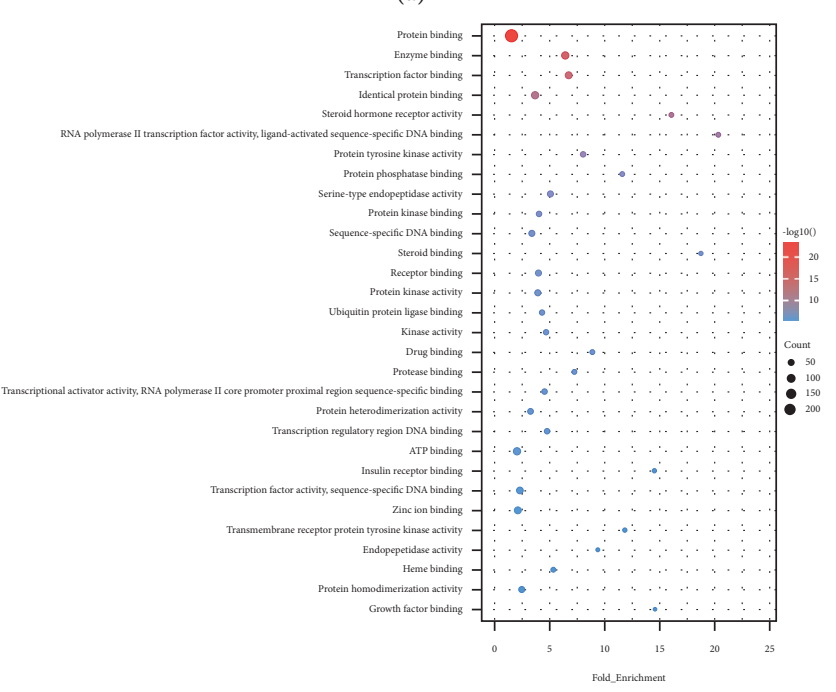

(c)

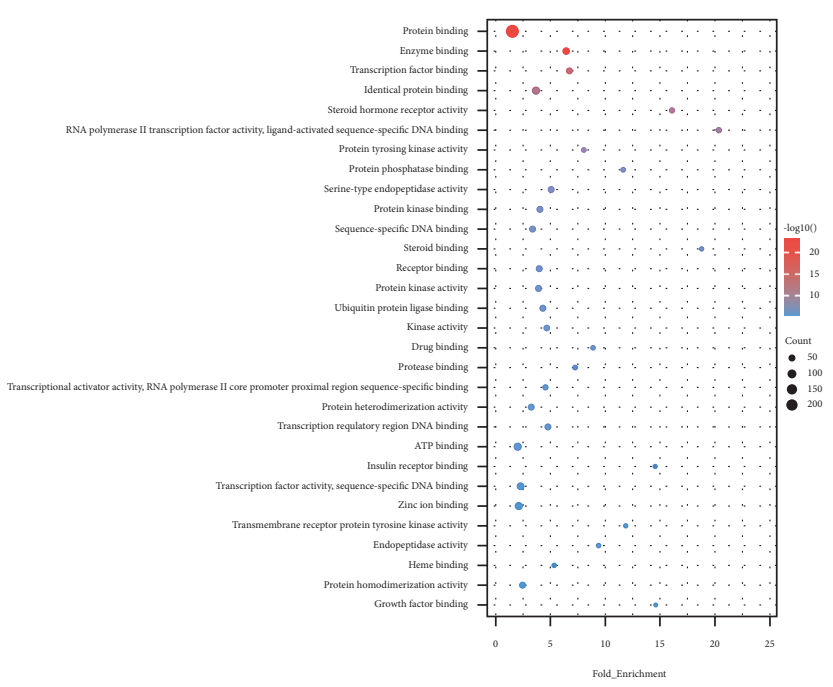

(b)

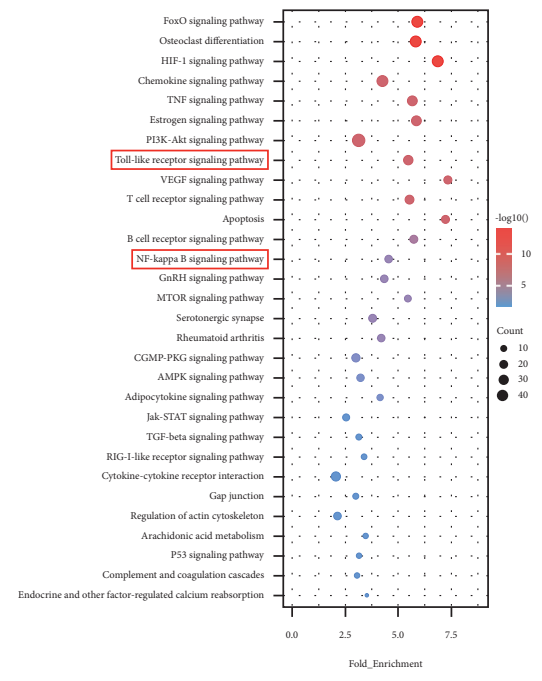

(d)

FIGURE 4: Bubble chart of enrichment analysis results. (a) biological processes; (b) cell components; (c) molecular function; (d) signaling pathways. $X$-axis stands for fold enrichment.

$(P<0.05)$. Compared with the model group, the expressions of TLR-4, MyD88, and NF- $\kappa \mathrm{B}$ p65 in the resveratrol lowdose and high-dose groups were significantly reduced $(P<0.05)$ (Figure 9).

3.6. Effect of Resveratrol on the Expression of Bcl-2, Bax, and Caspase-9 Protein. Compared with the blank control group, the expression of $\mathrm{Bcl}-2$ in the model group decreased $(P<0.05)$, and the expression of Bax and Caspase- 9 increased $(P<0.05)$. Compared with the model group, the expression of Bcl-2 in the resveratrol low-dose and highdose groups increased $(P<0.05)$, and the expression of Bax and Caspase-9 decreased $(P<0.05)$ (Figure 10$)$.

Through network pharmacological analysis, this study found that resveratrol was concentrated in the TLR4 signaling pathway, and animal experiments were used to verify the results. Experimental studies have shown that resveratrol can improve the degree of pathological damage in OA rats. Apoptosis is positively correlated with the severity of cartilage destruction and matrix depletion in human osteoarthritis tissue specimens. The Caspase family plays an important role in the process of cell apoptosis, and the excessive activation of Caspase- 9 and Caspase- 3 in the apoptotic cascade can promote cell apoptosis. The antiapoptotic member $\mathrm{Bcl} 2$ can prevent cell apoptosis, while the proapoptotic member Bax is located in the outer mitochondrial membrane or cytoplasm and oligomerizes under stress to promote the release of factors from mitochondria, thereby triggering apoptosis [52]. Burlacu found that the ratio of $\mathrm{Bcl}-2$ to $\mathrm{Bax}$ can reflect the relationship between $\mathrm{Bcl}$ 2 and Bax in the process of cell apoptosis: an increase in the ratio of $\mathrm{Bcl} 2 / \mathrm{Bax}$ inhibits cell apoptosis, and vice versa promotes cell apoptosis [53]. In this experiment, resveratrol can reduce the level of Caspase- 9 protein and increase the ratio of $\mathrm{Bcl}-2 / \mathrm{Bax}$. It shows that resveratrol inhibits the 


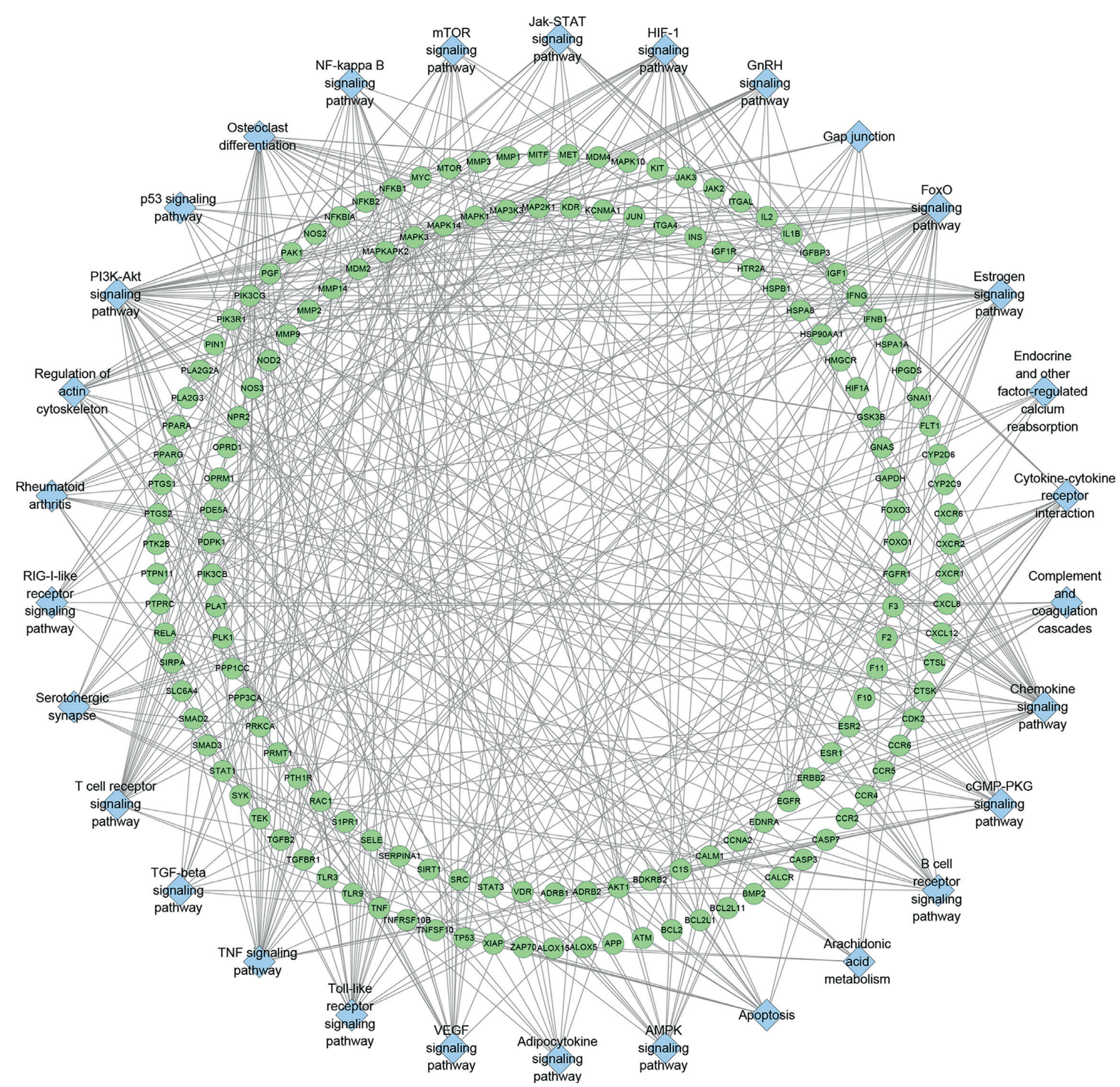

Figure 5: Signaling pathway-target network. Blue diamond stands for signaling pathway. Green circles stand for Resveratrol-OA targets.

apoptosis of chondrocytes in OA rats. In the pathology of bone and joint, proinflammatory cytokines play a key role. The levels of TNF- $\alpha$ and IL-6 are recognized cytokines that reflect the degree of inflammation. The high expression of IL- 6 can lead to the degradation of articular cartilage and induce pain. TNF- $\alpha$ is an inflammatory response mediator secreted by monocytes and macrophages. Long-term high TNF- $\alpha$ levels may be the main reason for the development of OA in wounded joints. IL-17 is a proinflammatory cytokine secreted by $\mathrm{T}$ lymphocytes and monocytes. MCP-1 is a representative of the $\beta$ subfamily of chemotactic cytokines and an important inflammatory response mediator of $\mathrm{OA}$ [54]. This study found that resveratrol can reduce the levels of IL- $1 \beta$, IL-6, IL17, TNF- $\alpha$, and MCP- 1 in the serum of OA rats. It shows that resveratrol can maintain the normal structure and function of joints by reducing the content of proinflammatory cytokines.

TLR4/myeloid differentiation factor 88 (My D88) signal transduction pathway is an inflammatory signal pathway that has been studied in the prevention and treatment of knee OA in recent years [55]. TLR4 is an important signal pathway transduction protein, which is closely related to the pathogenesis of OA. It is highly expressed in OA chondrocytes and participates in cartilage destruction. It can activate various inflammatory factors through its downstream MyD88-dependent signal transduction pathway [56]. $\mathrm{NF}-\kappa \mathrm{B}$ is a primary transcription factor that controls the expression of many proinflammatory genes and plays an important role in cell processes. Literature reports that plant extracts can affect the NF- $\kappa \mathrm{B}$ pathway [57]. Studies have 


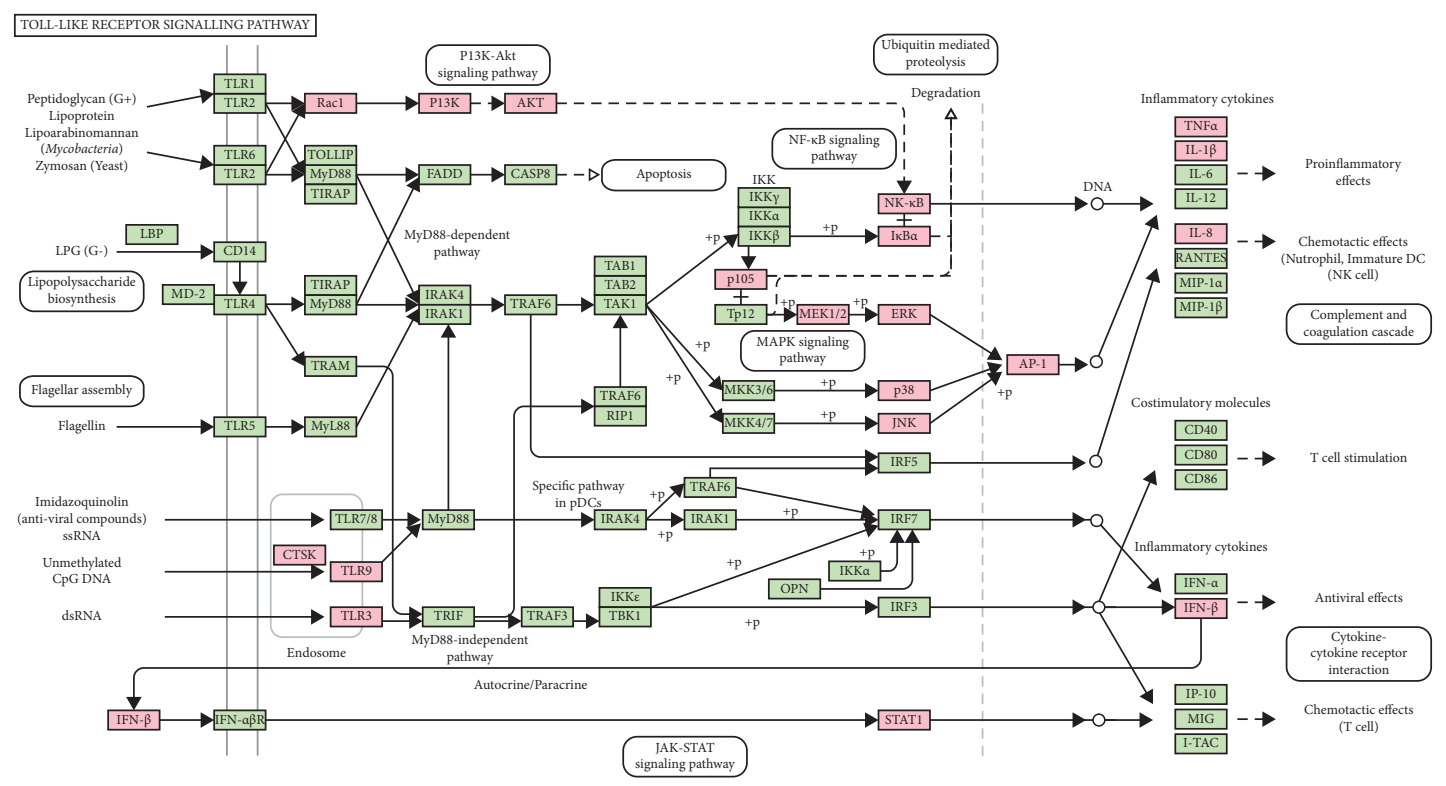

(a)

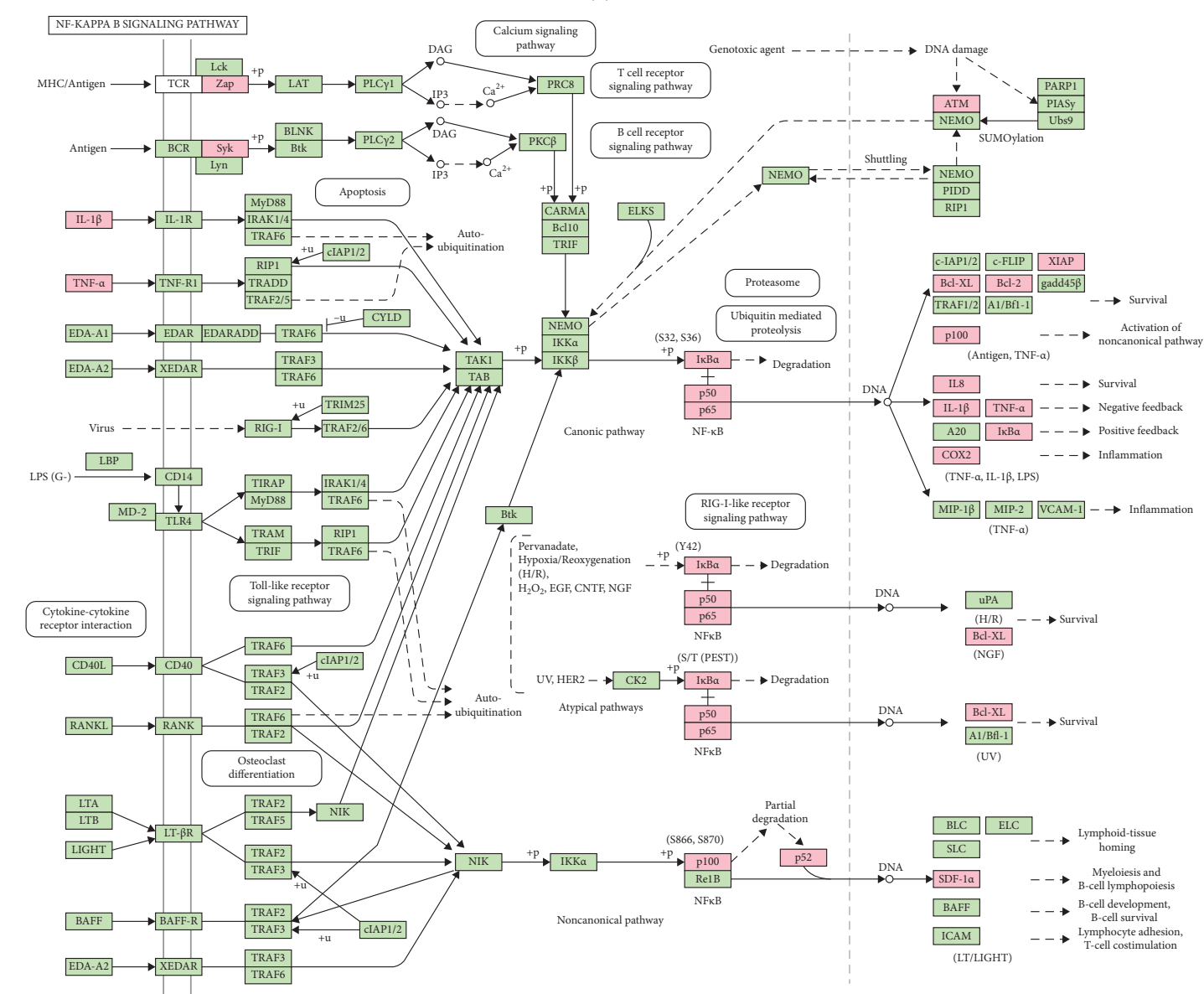

$040644 / 12 / 19$

(c) Kanehisa Laboratories

(b)

FIGURE 6: (a) Toll-like receptor signaling pathway adapted from KEGG (ID: hsa04620); (b) NF-kB signaling pathway adapted from KEGG (ID: hsa04064). 


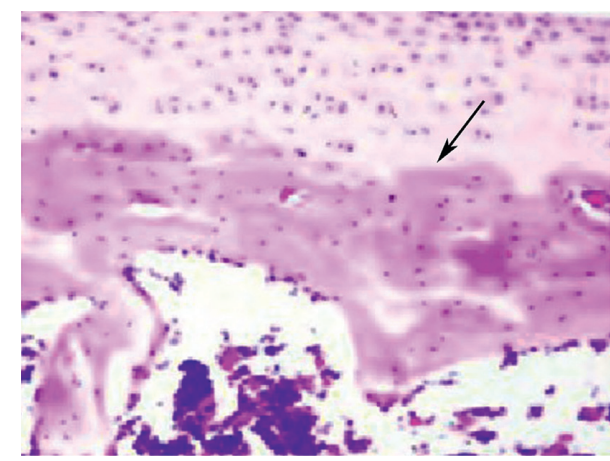

(a)

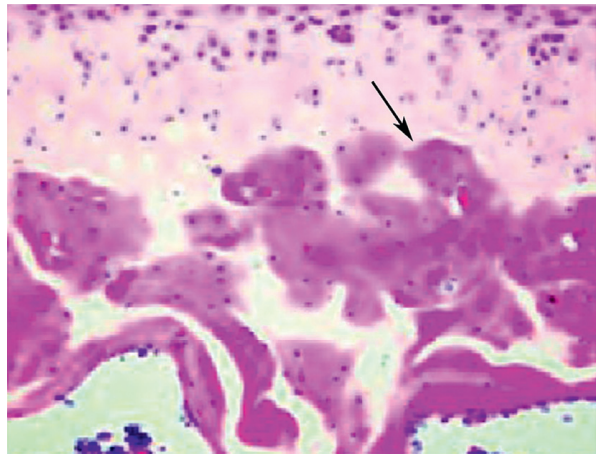

(c)

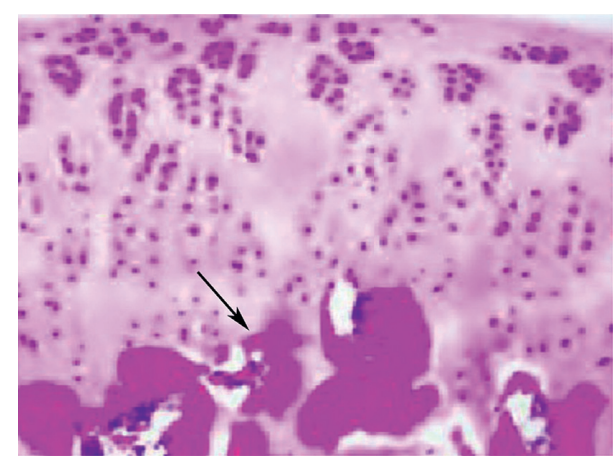

(b)

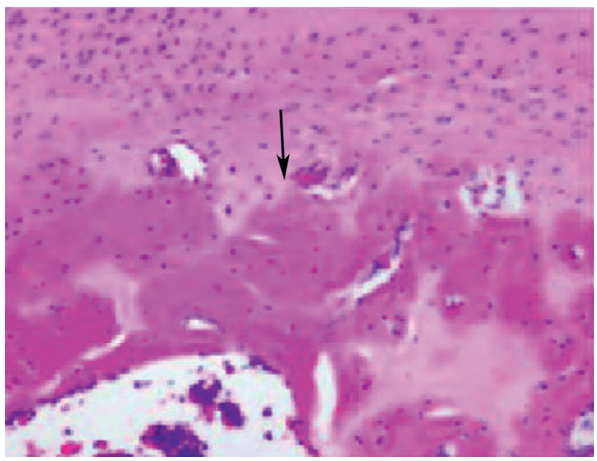

(d)

Figure 7: Pathological changes (HE staining; X200). (a) Control group; (b) model group; (c) resveratrol low-dose group; (d) resveratrol high-dose group. The black arrow indicates tidemark.

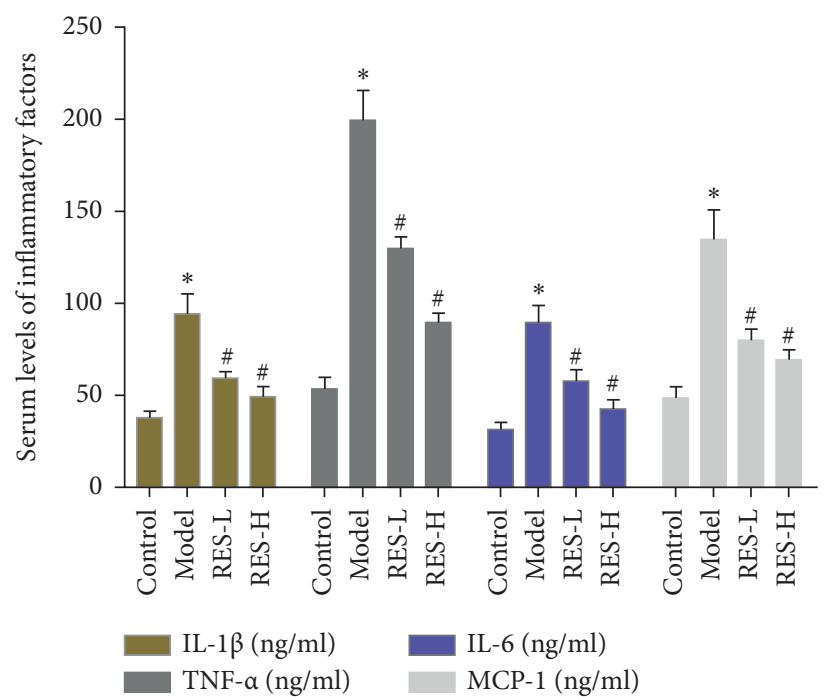

FIgURE 8: Effect of Resveratrol on Serum IL-1 $\beta$, IL-6, TNF- $\alpha$, MCP-1 Content (RES-L: resveratrol low-dose group; RES-H: resveratrol highdose group; * compared with the control group, $P<0.05$; ${ }^{*}$ compared with model group, $P<0.05$ ).

reported that the use of siRNA to interfere with the expression of $\mathrm{NF} \kappa \mathrm{B}$ p 65 can reduce the pathological process of $\mathrm{OA}$ in rats, indicating that inhibiting the activity of $\mathrm{NF}-\kappa \mathrm{B}$ can be used as a target for the treatment of osteoarthritis [58]. This study found that resveratrol downregulated the protein levels of TLR-4, MyD88 and the ratio of $\mathrm{pNF}-\kappa \mathrm{B}$ p65/NF- $\kappa \mathrm{B}$ p65 in osteoarthritis rats. This shows that resveratrol can treat $\mathrm{OA}$ rats by inhibiting TLR-4/MyD88 and $\mathrm{NF}-\kappa \mathrm{B}$ signaling pathways.

In summary, resveratrol improves the degree of pathological damage in rats with bone OA, reduces cartilage tissue apoptosis, increases the proportion of trabecular bone and the proportion of cartilage, inhibits the degradation of extracellular matrix, promotes the synthesis of extracellular 


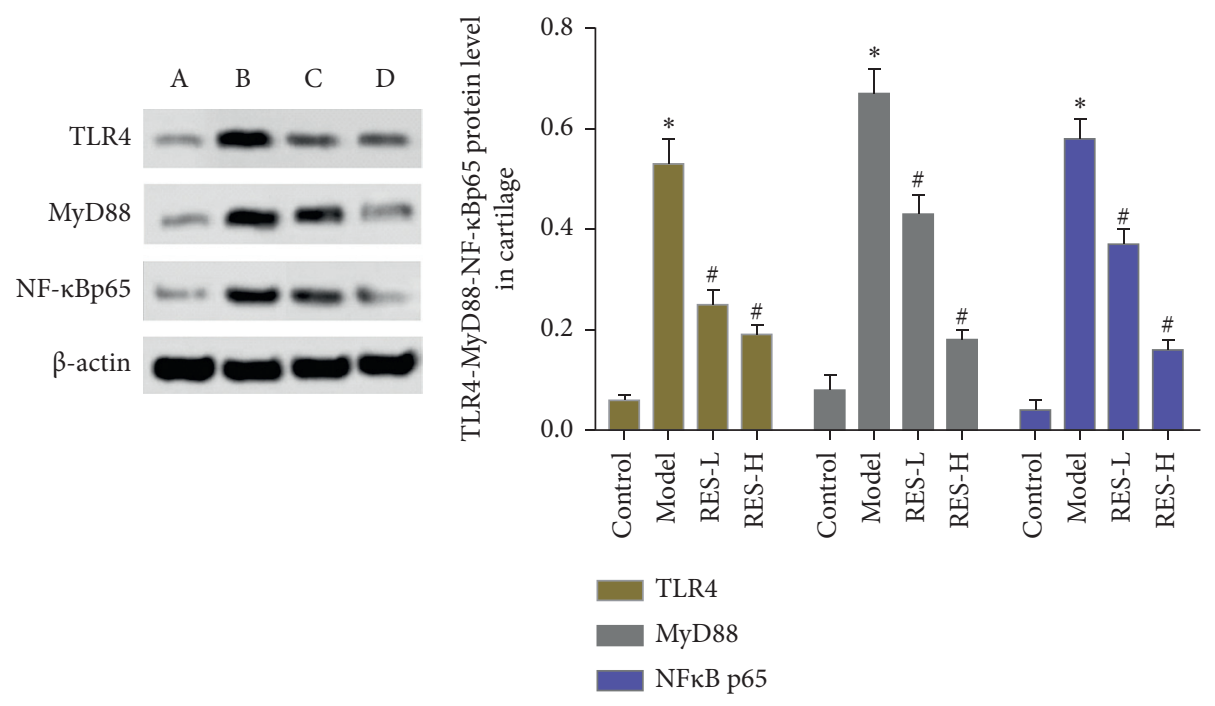

Figure 9: Effect of resveratrol on the expression of TLR-4, MyD88, NF- $\kappa$ B p65 protein (A) control group; (B) model group; (C) resveratrol low-dose group; (D) resveratrol high-dose group. ${ }^{*}$ Compared with the control group, $P<0.05$; ${ }^{*}$ compared with model group, $P<0.05$.
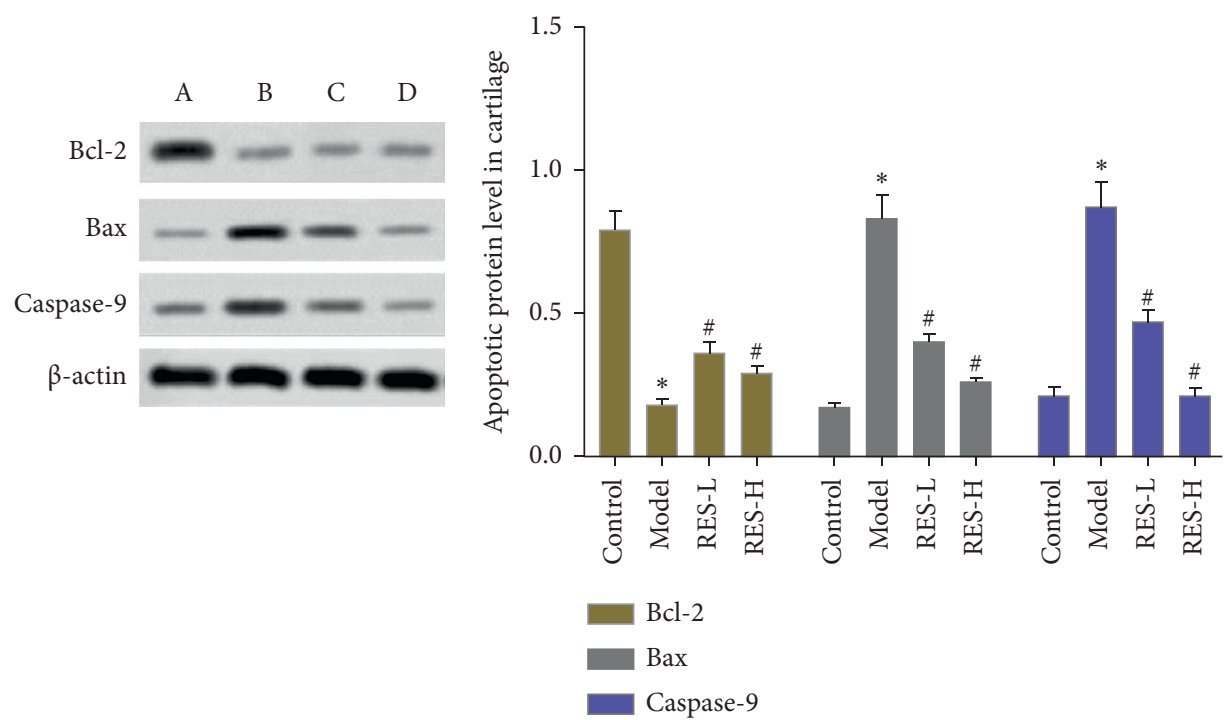

Figure 10: Effect of resveratrol on the expression of Bcl-2, Bax, Caspase-9 protein; (A) control group; (B) model group; (C) resveratrol lowdose group; (D) resveratrol high-dose group. ${ }^{*}$ Compared with control group, $P<0.05$; \#compared with model group, $P<0.05$.

matrix, and reduces the content of proinflammatory cytokines. This may be achieved through the NF- $\kappa \mathrm{B}$ signaling pathway mediated by TLR4. This study provides a theoretical basis for the clinical application of resveratrol in the treatment of OA.

\section{Conclusion}

Resveratrol may inhibit the activation of the TLR4-mediated $\mathrm{NF}-\kappa \mathrm{B}$ signaling pathway and has a repairing effect on soft tissue damage in OA.

\section{Data Availability}

The data that support the findings of this study are available in supplementary materials.

\section{Disclosure}

Zhiyong Long, Wang Xiang, and Ganpeng Yu are considered as joint first authors.

\section{Conflicts of Interest}

The authors declare that there are no conflicts of interest.

\section{Authors' Contributions}

Zhiyong Long, Wang Xiang, Ganpeng Yu, Jun Li, and Tiejun Yang are responsible for the study concept and design. Zhiyong Long, Wang Xiang, Ganpeng Yu, Jun Li, and Tiejun Yang are responsible for the data collection, data analysis, and interpretation. Zhiyong Long and Wang Xiang drafted 
the paper; Q Ganpeng Yu, Jun Li, and Tiejun Yang supervised the study; all the authors participated in the analysis and interpretation of data and approved the final paper.

\section{Acknowledgments}

This research was supported by Hunan Province Clinical Medical Technology Innovation Guidance Project (No. 2018SK50235).

\section{Supplementary Materials}

Table S1: Resveratrol Potential Targets. Table S2: OA genes. Table S3: Enrichment Analysis of Resveratrol-OA PPI Network. (Supplementary Materials)

\section{References}

[1] J. B. SnellingS, S. Bas, and J. PuskasG, "Presence of IL-17 in synovial fluid identifes a potential inflammatory osteathitic phenotype," PloS One, vol. 12, no. 4, 2017.

[2] D. T. Felson, A. Naimark, J. Anderson, L. Kazis, W. Castelli, and R. F. Meenan, "The prevalence of knee osteoarthritis in the elderly. the framingham osteoarthritis study," Arthritis \& Rheumatism, vol. 30, no. 8, pp. 914-918, 1987.

[3] J. L. Van Saase, L. K. Van Romunde, A. Cats, J. P. Vandenbroucke, and H. A. Valkenburg, "Epidemiology of osteoarthritis: zoetermeer survey. Comparison of radiological osteoarthritis in a Dutch population with that in 10 other populations," Annals of the Rheumatic Diseases, vol. 48, no. 4, pp. 271-280, 1989.

[4] G. Peat, R. McCamney, and P. Croft, "Knee pain and osteoarthritis in older adults: a review of community burden and current use of primary health care," Annals of the Rheumatic Diseases, vol. 60, no. 2, pp. 91-97, 2001.

[5] L. Busija, L. Bridgett, S. R. M. Williams, R. Buchbinder, L. March, and M. Fransen, "Osteoarthritis," Best Practice \& Research Clinical Rheumatology, vol. 24, no. 6, pp. 757-768, 2010.

[6] I. Osborne, C. K. Kwoh, A. Guermazi et al., "Synovitis in knee osteoarthritis: a precursor of disease?" Annals of the Rheumatic Diseases, vol. 75, no. 2, pp. 390-395, 2016.

[7] K. D 'Lima, D. D. D’Lima, S. Hashimoto, and M. Lotz, "Cell death in cartilage," Osteoarthritis and Cartilage, vol. 12, no. 1, pp. 1-16, 2004.

[8] M. Kapoor, J. Martel-Pelletier, D. Lajeunesse, J. P Pelletier, and H Fahmi, "Role of proinflammatory cytokines in the pathophysiology of osteoarthritis," Nature Reviews. Rheumatology, vol. 7, no. 1, pp. 33-42, 2011.

[9] O. Bruyere, C. Cooper, and N. Arden, "Can we identify patients with high nisk of osteoathrtisprogresson who will resp ond to treatment? A focus on epidemiology and phenotype of osteoarthritis," Drugs \& Aging, vol. 32, no. 3, pp. 179-187, 2015.

[10] D. Huang, Q. Zhao, H. Liu, Y. Guo, and H. Xu, "PPAR- $\alpha$ agonist WY-14643 inhibits LPS-induced inflammation in synovial fibroblasts via NF-kB pathway," Journal of Molecular Neuroscience, vol. 59, no. 4, pp. 544-553, 2016.

[11] P. A. Dieppe and L. S. Lohmander, "Pathogenesis and management of pain in osteoarthritis," The Lancet, vol. 365, no. 9463, pp. 965-973, 2005.

[12] G. A. Hawker, "Osteoarthritis is a serious disease," Clinical \& Experimental Rheumatology, vol. 37, no. 5, pp. 3-6, 2019.

[13] A. E. M. Jargensen, M. Kjer, and K. M. Heinemeier, "The effect of A ging and mechanical loading on the metabolism of articular cartilage," The Joumnal of Rheumatology, vol. 44, no. 4, pp. 410-417, 2017.

[14] J. A. Martin and J. A. Buckwalter, "Aging, articular cartilage chondrocytesenescence and osteoarthritis," Biogerontology, vol. 3, no. 5, pp. 257-264, 2002.

[15] X. Liang, H. Li, and S. Li, "A novel network pharmacology approach to analyse traditional herbal formulae: the Liu-WeiDi-Huang pill as a case study," Molecular BioSystems, vol. 10, no. 5, pp. 1014-1022, 2014.

[16] J. Gu and S. Li, "Towards integrative annotation of the celltype specific gene functional and signaling map in vascular endothelial cells," Molecular BioSystems, vol. 8, no. 8, pp. 2041-2049, 2012.

[17] S. Li, T. P. Fan, W. Jia, A. Lu, and W. Zhang, "Network pharmacology in traditional Chinese medicine," Evidencebased Complementary and Alternative Medicine, vol. 2014, Article ID 138460, 2 pages, 2014.

[18] D. Gfeller, A. Grosdidier, M. Wirth, A. Daina, O. Michielin, and V. Zoete, "SwissTargetPrediction: a web server for target prediction of bioactive small molecules," Nucleic Acids Research, vol. 42, no. W1, pp. W32-W38, 2014.

[19] X. F. Liu, S. S. Ouyang, and Y. Biao, "PharmMapper server: a web server for potential drug target identification using pharmacophore mapping approach," Nucleic Acids Research, vol. 38, pp. W609-W614., 2017.

[20] M. J. Keiser, B. L. Roth, B. N. Armbruster, P. Ernsberger, J. J. Irwin, and B. K. Shoichet, "Relating protein pharmacology by ligand chemistry," Nature Biotechnology, vol. 25, no. 2, pp. 197-206, 2007.

[21] M. Kuhn, D. Szklarczyk, A. Franceschini et al., "Stitch 2: an interaction network database for small molecules and proteins," Nucleic Acids Research, vol. 38, no. suppl_1, pp. D552-D556, 2010.

[22] S. Kim, J. Chen, T. Cheng et al., "PubChem 2019 update: improved access to chemical data," Nucleic Acids Research, vol. 47, no. D1, pp. D1102-D1109, 2019.

[23] G. Stelzer, R. Rosen, I. Plaschkes et al., "The GeneCards suite: from gene data mining to disease genome sequence analysis," Current Protocols in Bioinformatics, vol. 54, pp. 1301-1303, 2016.

[24] A. Hamosh, A. F. Scott, and J. S. Amberger, "Online Mendelian Inheritance in Man (OMIM), a knowledgebase of human genes and genetic disorders," Nucleic Acids Research, vol. 33, pp. D514-D517, 2014.

[25] D. Szklarczyk, A. Franceschini, S. Wyder et al., "STRING v10: protein-protein interaction networks, integrated over the tree of life," Nucleic Acids Research, vol. 43, no. D1, pp. D447-D452, 2015.

[26] D. W. Huang, B. T. Sherman, and R. A. Lempicki, "Systematic and integrative analysis of large gene lists using DAVID Bioinformatics Resources," Nature Protocols, vol. 4, no. 1, pp. 44-57, 2009.

[27] X. Gao, D. Deeb, J. Media et al., "Immunomodulatory activity of resveratrol: discrepant in vitro and in vivo immunological effects," Biochemical Pharmacology, vol. 66, no. 12, pp. 2427-2435, 2003.

[28] Y. Wei, J. Jia, X. Jin, W. Tong, and H. Tian, "Resveratrol ameliorates inflammatory damage and protects against osteoarthritis in a rat model of osteoarthritis," Molecular Medicine Reports, vol. 17, no. 1, pp. 1493-1498, 2018.

[29] M. Kanehisa, Y. Sato, and M. Kawashima, "KEGG mapping tools for uncovering hidden features in biological data," Protein Science, 2021.

[30] F. K. Nielsen, N. Egund, A. Jørgensen, and A. G. Jurik, "Risk factors for joint replacement in knee osteoarthritis; a 15-year 
follow-up study," BMC Musculoskeletal Disorders, vol. 18, no. 1 , p. $510,2017$.

[31] W. Hideto, "Aggrecan and its chondroitin sulfate in cartilage," Seikagaku, vol. 80, no. 1, p. 28, 2008.

[32] L. Pulsatelli, O. Addimanda, V. Brusi, B. Pavloska, and R. Meliconi, "New findings in osteoarthritis pathogenesis: therapeutic implications," Therapeutic Advances in Chronic Disease, vol. 4, no. 1, pp. 23-43, 2013.

[33] C. B. Little, A. Barai, D. Burkhardt et al., "Matrix metalloproteinase 13â"deficient mice are resistant to osteoarthritic cartilage erosion but not chondrocyte hypertrophy or osteophyte development," Arthritis \& Rheumatism, vol. 60, no. 12, pp. 3723-3733, 2009.

[34] T. Sato, K. Konomi, S. Yamasaki et al., "Comparative analysis of gene expression profiles in intact and damaged regions of human osteoarthritic cartilage," Arthritis \& Rheumatism, vol. 54, no. 3, pp. 808-817, 2006.

[35] D. Philipot, D. Guérit, D. Platano et al., "p16INK4a and its regulator miR-24 link senescence and chondrocyte terminal differentiation-associated matrix remodeling in osteoarthritis," Arthritis Research and Therapy, vol. 16, no. 1, p. R58, 2014.

[36] H. Li, D. Wang, Y. Yuan, and J. Min, "New insights on the MMP-13 regulatory network in the pathogenesis of early osteoarthritis," Arthritis Research and Therapy, vol. 19, no. 1, p. 248, 2017.

[37] Y. Wan, W. Li, Z. Liao, M. Yan, X. Chen, and Z. Tang, "Selective MMP-13 inhibitors: promising agents for the therapy of osteoarthritis," Current Medicinal Chemistry, vol. 27, no. 22, pp. 3753-3769, 2020.

[38] C. Csali, N. Kes his hzadeh, and K. Fise her, "Regulation of iflamnatnonsigalling by resveratrol in $\mathrm{ku}$ man $\mathrm{c}$ hondrocytes in vntro," Biochemical phaIaology, vol. 75, no. 3, pp. 677-687, 2008.

[39] M. Shakibaei, C. Csaki, S. Nebrich, and A. Mobasheri, "Resveratrol suppresses interleukin- $1 \beta$-induced inflammatory signaling and apoptosis in human articular chondrocytes: potential for use as a novel nutraceutical for the treatment of osteoarthritis," Biochemical Pharmacology, vol. 76, no. 11, pp. 1426-1439, 2008.

[40] E. Limagne, A. Lancon, and D. Delmas, "Resveratrol interferes with IL 1-b eta -induced pro -inflammatory paracrine interacti on between primary chondrocytes and macrophages," Nutrients, no. 5, p. 8, 2016.

[41] L. Liu, H. Gu, H. Liu et al., "Protective effect of resveratrol against IL-1 $\beta$-induced inflammatory response on human osteoarthritic chondrocytes partly via the TLR4/MyD88/NF- $\kappa \mathrm{B}$ signaling pathway: an "in vitro study"," International Journal of Molecular Sciences, vol. 15, no. 4, pp. 6925-6940, 2014.

[42] K. Bobacz, I. G. Sunk, J. G. Hofstaetter et al., "Toll-like receptors and chondrocytes: the lipopolysaccharide-induced decrease in cartilage matrix synthesis is dependent on the presence of toll-like receptor 4 and antagonized by bone morphogenetic protein 7," Arthritis \& Rheumatism, vol. 56, no. 6, pp. 1880-1893, 2007.

[43] H. Gu, Y. Jiao, and X. Yu, "Resveratrol inhibits the IL- $1 \mathrm{~b}$ eta -induced expression of MMP-13 and IL-6 in human articular chondrocytes via TLR4/MyD 88-dependent and-independent signaling ca sca des," Intermational journal of molecular medi cine, 2017.

[44] J. Y. Lee and G. Z. YeJ, "Reciproalmodulation of Tl-1ke receptor-4 signaling pathways inv olvingMyD 88 and pho sphatidylinositol 3-kinase/AKT by saturated and polyunsaturated fatty acids," Journal of Biological Chemistry, vol. 278, no. 39, pp. 3704 1-37051, 2003.
[45] E. Aksamitiene, B. N. Kiyatkin, and B. N. Kholodenko, "Cross-talk between mitogenic Ras/MAPK and survival PI3K/ Akt pathways: a fine balance," Biochemical Society Transactions, vol. 40, no. 1, pp. 139-146, 2012.

[46] M. Y. Zhu, J. L. Guo, and H. Xia, "[The anti-apoptotic effect of cytoplasmic alpha- fetoprotein in hepatoma cells induced by all trans retinoic acid involves activation of the PI3K/AKT signaling pathway]," Zhonghuaganzangbing za zhi= Zhonghuaganzangbingzazhi $=$ Chinese joumalofhepatology, vol. 22, no. 1 1, pp. 837-842, 2014.

[47] R. J. Atkins, L. Dimou, A. P. Morokoff, A. H. Kaye, K. J. Drummond, and C. M. Hovens, "Regulation of glycogen synthase kinase- 3 beta (GSK-3 $\beta$ ) by the Akt pathway in gliomas," Journal of Clinical Neuroscience, vol. 19, no. 11, pp. 1558-1563, 2012.

[48] Y. Sun, C. Wu, J. Ma et al., "Toll-like receptor 4 promotes angiogenesis in pancreatic cancer via PI3K/AKT signaling," Experimental Cell Research, vol. 347, no. 2, pp. 274-282, 2016.

[49] D. Xu, Y. Li, B. Zhang et al., "Resveratrol alleviate hypoxic pulmonary hypertension via anti-inflammation and antioxidant pathways in rats," International Journal of Medical Sciences, vol. 13, no. 12, pp. 942-954, 2016.

[50] A. M. Th omp son, K. A. Martin, and E. M. Rzucidlo, "Resveratro1 induces vascularsmooth musclecell differentiati on through stimulation of SirT1 and AMPK," P1oS one, vol. 9, no. 1, pp. e8-95, 2014.

[51] Y. Zong, L. Sun, B. Liu et al., "Resveratrol inhibits LPS-induced MAPKs activation via activation of the phosphatidylinositol 3-kinase pathway in murine RAW 264.7 macrophage cells," PloS One, vol. 7, no. 8, Article ID e44107, 2012.

[52] P. E. Czabotar, G. Lessene, A. Strasser, and J. M. Adams, "Control of apoptosis by the bcl-2 protein family: implications for physiology and therapy," Nature Reviews Molecular Cell Biology, vol. 15, no. 1, pp. 49-63, 2014.

[53] A. Burlacu, "Regulation of apoptosis by bcl-2 family proteins," Journal of Cellular and Molecular Medicine, vol. 7, no. 3, pp. 249-257, 2010.

[54] H. M. Ismail, K. Yamamoto, T. L. Vincent, H. Nagase, L. Troeberg, and J. Saklatvala, "Interleukin-1 acts via the jnk-2 signaling pathway to induce aggrecan degradation by human chondrocytes," Arthritis \& Rheumatology, vol. 67, no. 7, pp. 1826-1836, 2015.

[55] J. Liu, C. Z. Qu, and P. J. Xie, "Changes in cartilage Toll-like receptor 4/myeloid differentiation factor 88 signal transduction pathway in knee osteoarthritis model rats after receiving massage treatment," Chinese Tissue Engineering Research, vol. 19, pp. 3019-3024, 2019, [in chinese].

[56] F. Hua, T. Ha, J. Ma et al., "Blocking the MyD88-dependent pathway protects the myocardium from ischemia/reperfusion injury in rat hearts," Biochemical and Biophysical Research Communications, vol. 338, no. 2, pp. 1118-1125, 2005.

[57] Y. Ding, S. Yuan, X. Liu et al., "Protective effects of astragaloside IV on $\mathrm{db} / \mathrm{db}$ mice with diabetic retinopathy," PloS One, vol. 9, no. 11, Article ID e112207, 2014.

[58] L. X. Chen, L. Lin, H. J. Wang et al., "Suppression of early experimental osteoarthritis by in vivo delivery of the adenoviral vector-mediated NF- $\kappa$ Bp65-specific siRNA," Osteoarthritis and Cartilage, vol. 16, no. 2, pp. 174-184, 2008. 